\title{
Activation of cannabinoid receptor type 2- induced osteogenic differentiation involves autophagy induction and p62-mediated Nrf2 deactivation
}

\author{
Aihua Xu, Yang Yang, Yang Shao, Meng Wu and Yongxin Sun ${ }^{*}$
}

\begin{abstract}
Background: Dysfunction in survival and differentiation of osteoblasts commonly occurs in patients with osteoporosis. Cannabinoid receptor type 2 (CNR2) is a major receptor of endocannabinoid system that is crucial for bone mass homeostasis. Our group prior demonstrated that activation of CNR2 signaling promoted osteogenic differentiation of bone marrow derived mesenchymal stem cells in vitro. Autophagy is reported to participate in osteoblastic differentiation. Whether autophagy is regulated by CNR2-mediated cannabinoid signaling is unknown, and how the autophagy-CNR2 interaction affects osteoblastic differentiation requires further elucidation.

Methods: hFOB 1.19 osteoblasts were treated with CNR2 agonists HU308 (5, 10, 25, 50 or 100 nM) and JWH133 (1, 2, 5, 10 or $20 \mu \mathrm{M}$ ) in presence or absence of autophagy inhibitor 3-Methyladenine (3-MA). The differentiation of hFOB 1.19 cells was determined via evaluating their alkaline phosphatase (ALP) activity and mineralization ability (Alizarin red staining). Alterations in autophagy-related molecules and osteogenic markers were analyzed via real-time PCR and/or immunoblotting assays.

Results: hFOB 1.19 cells spontaneously differentiated towards mature osteoblasts under $39^{\circ} \mathrm{C}$, during which CNR2 expression increased, and autophagy was activated. The strongest autophagy flux was observed at $192 \mathrm{~h}$ post differentiation-LC3I to LC3II conversion was enhanced and Beclin 1 expression was upregulated considerably, while p62 expression was downregulated. Treatment of HU308 and JWH133 promoted autophagy in a dose-dependent manner, and suppressed mTOR signaling pathway in hFOB 1.19 cells. In CNR2-silenced cells, HU308's and JWH133's effects on autophagy were weakened. HU308 and JWH133 enhanced the ALP activity and mineralization, and upregulated the expression of osteogenic markers, osteopontin and osteocalcin, in hFOB 1.19 cells. Intriguingly, such pro-osteogenic effects induced by CNR2 activation were markedly mitigated by 3-MA. In addition to provoking autophagy, CNR2 agonists also reduced nuclear Nrf2 accumulation and increased Keap1 expression. Further, reexpression of p62 inhibited CNR2 agonists-induced Nrf2 degradation.
\end{abstract}

Conclusions: Osteogenic differentiation induced by CNR2 signaling activation involves autophagy induction and p62mediated Nrf2 deactivation.

Keywords: Osteoporosis, Osteoblast, CNR2, Autophagy, p62, Nrf2 signaling pathway

\footnotetext{
*Correspondence: sunyongxin@cmu.edu.cn

Department of Rehabilitation Medicine, The First Affiliated Hospital of China

Medical University, 155 North Nanjing Street, Shenyang, Liaoning 110001,

People's Republic of China
}

(c) The Author(s). 2020 Open Access This article is distributed under the terms of the Creative Commons Attribution 4.0 International License (http://creativecommons.org/licenses/by/4.0/), which permits unrestricted use, distribution, and reproduction in any medium, provided you give appropriate credit to the original author(s) and the source, provide a link to the Creative Commons license, and indicate if changes were made. The Creative Commons Public Domain Dedication waiver (http://creativecommons.org/publicdomain/zero/1.0/) applies to the data made available in this article, unless otherwise stated. 


\section{Background}

Osteoporosis is a systemic skeletal disorder featured by structural deterioration of bone mass and reduction of bone mineral density, and it is a silent disease until a fracture occurs [1, 2]. Excessive use of glucocorticoids [3] and deficiency of estrogen [4] are two major clinical risk factors that augment the risk of fragility fracture in aged population and postmenopausal women, respectively. Dysfunction in survival and differentiation of osteoblasts, a cell population responsible for generating extracellular matrix proteins and regulating matrix mineralization, is commonly found in patients with osteoporosis [5]. Hence, osteoblasts are considered as a target of anti-osteoporotic therapy [6].

Cannabinoid receptor type 2 (CNR2) is a G proteincoupled receptor that binds to endogenous cannabinoids [7]. By giving osteoporotic animals with synthetic CNR2 agonists or antagonists, several previous studies revealed a conflicting role of CNR2-mediated cannabinoid signaling in regulating the homeostasis of bone mass. Idris and coworkers proved that AM630, a CNR2-selective antagonist, mitigated ovariectomy-induced bone loss in mice [8]. This team further demonstrated that mice lacking CNR1 and CNR2 had higher trabecular bone mass and displayed resistance to ovariectomy-induced osteoporosis [9]. They deduced that the negative regulatory role of activated CNR2 signaling in bone formation was associated with enhanced osteoclast formation. Ofek and colleagues, on the contrary, demonstrated that CNR2-deficient mice had significant age-related trabecular bone loss [10]. They also found that HU308, a CNR2-selective agonist, mitigated ovariectomy-induced bone loss [10]. Our prior work showing that activation of CNR2 signaling induced by UR144 augmented the osteogenic differentiation of bone marrow-derived mesenchymal stem cells (BMMSCs) [11] supported an osteogenesis-promoting role of CNR2. We here further elucidated the underlying mechanisms with a focus on autophagy and Nuclear Factor Erythroid 2 Related Factor 2 (Nrf2) signaling pathway.

Cells often initiate autophagy to deliver damaged organelles or protein aggregates to vacuole or lysosome where the cargos are degraded and recycled [12]. Recently, activation of autophagy is reported to enhance the osteogenic differentiation. The mineralization of BMMSCs was suppressed when autophagy was inhibited, whereas enhanced when autophagy was re-activated [13]. How CNR2 signaling transduction affects autophagy during osteogenic differentiation is unclear. Nevertheless, several previous lines of evidence imply that CNR2 agonists can regulate autophagy flux. In macrophages and cardiomyocytes, HU308 was found to enhance cellular autophagy $[14,15]$. We therefore proposed that activation of CNR2 signaling may enhance the osteogenic differentiation by provoking autophagy.
Under quiescent condition, Kelch-like ECH-associated protein 1 (Keap1), binds to Nrf2, a transcription factor, and induces its constitutive degradation through the ubiquitin-proteasome pathway [16, 17]. Keap1-deficient mice exhibited hyperactivation of Nrf2 and had impaired bone formation [18]. Such negative regulatory role of over-activated Nrf2 is suggested to link to its inhibitory effects on osteoblast differentiation [19]. The Sequestosome 1 (SQSTM1)/p62 (hereafter referred to p62) is a common component of protein aggregates, and it can be recruited to ubiquitinated protein aggregates [20, 21]. During dynamic autophagy, p62 functions as a bridge between autophagosomes and polyubiquitinated cargo, and p62 itself eventually becomes degraded [22]. Interestingly, p62 contains a Keap1-interacting region (KIR) similar to Nrf2, which enables p62 to tone up Nrf2 signaling transduction through competitively binding to Keap1 [23]. We here also investigate whether CNR2 agonists affect Nrf2 signaling transduction, and whether p62 is involved in such process.

Herein, hFOB 1.19 osteoblasts were treated with two synthetic CNR2 ligands, HU308 and JWH133, and their differentiation was determined by analyzing the cell mineralization, activity of alkaline phosphatase (ALP) and expression of bone formation markers. We found that osteogenic differentiation induced by CNR2 activation was inhibited when autophagy was blocked with 3-MA. CNR2 agonists reduced Nrf2 expression in hFOB 1.19 cells by accelerating p62 degradation.

\section{Materials and methods}

\section{Cell culture and RNA interference (RNAi) of CNR2}

Human osteoblastic hFOB 1.19 cells (Procell, Wuhan, China) were routinely maintained in DMEM/F12 medium (Procell) containing 10\% fetal bovine serum (FBS; Sigma-Aldrich, St. Louis MO, USA) and $0.3 \mathrm{mg} /$ ml G418 under $5 \% \mathrm{CO}_{2}$ and $95 \%$ air at $34{ }^{\circ} \mathrm{C}$. hFOB 1.19 cells were cultured at $34^{\circ} \mathrm{C}$ until reaching confluence, and then transferred to $39^{\circ} \mathrm{C}$ as described previously [24]. After being cultured at $39^{\circ} \mathrm{C}$ for $48 \mathrm{~h}$, cells were further treated with $\operatorname{HU} 308(5,10,25,50$ or $100 \mathrm{nM}$; purity $\geq 98 \%$ determined with HPLC; Tocris Bioscience, Minneapolis, MN, USA) or JWH133 (1, 2, 5, 10 or $20 \mu \mathrm{M}$; purity $\geq 98 \%$ determined with HPLC; Tocris Bioscience) for additional $12 \mathrm{~h}, 96 \mathrm{~h}$ or $192 \mathrm{~h}$. As $100 \mathrm{nM}$ HU308 and $20 \mu \mathrm{M}$ JWH133 contained 0.05\% DMSO, to eliminate the effects induced by the solvent, all cell culture media contained 0.05\% DMSO.

CNR2 short hairpin RNA (CNR2 shRNA-S, 5' ttcatcaactccatggtcaattcaagagattgaccatggagttgatgattttttc $3{ }^{\prime}$; $\mathrm{CN}$ R2 shRNA-AS, 5' tcgagaaaaaatcatcaactccatggtcaatctcttgaattgaccatggagttgatgaa 3') was inserted into pSico lentiviral shuttle plasmid (Addgene,Cambridge, MA, USA) between $\mathrm{HpaI}$ and XhoI sites. Then pSico-CNR2 shRNA 
plasmid, psPAX2 package plasmid and pMD2.G envelope plasmid were co-transfected into $293 \mathrm{~T}$ cells to generate CNR2 shRNA lentiviruses (LV-CNR2 shRNA). hFOB 1.19 cells were infected with LV-CNR2 shRNA at $34{ }^{\circ} \mathrm{C}$, and $24 \mathrm{~h}$ later, cells were transferred to $39^{\circ} \mathrm{C}$ for differentiation. Forty-eight hours later, cells were further treated with $50 \mathrm{nM} \mathrm{HU} 308$ or $10 \mu \mathrm{M}$ JWH133 for $12 \mathrm{~h}$, and then harvested.

Human BMMSCs obtained from ZqxzBio (Shanghai, China) were cultured in MSC specific culture medium according to the supplier's protocols. Osteogenic differentiation of BMMSCs was induced according to our prior work [11]. BMMSCs were cultured in osteoinductive media with or without $50 \mathrm{nM}$ HU308 and $10 \mu \mathrm{M}$ JWH133. BMMSCs were infected with LV-CNR2 shRNA or control lentiviruses, and $24 \mathrm{~h}$ later, they were incubated in osteoinductive media for $48 \mathrm{~h}$. Then, BMMSCs were stimulated with $50 \mathrm{nM}$ HU308 and $10 \mu \mathrm{M}$ JWH133 for $12 \mathrm{~h}$, and then collected.

\section{Alkaline phosphatase (ALP) activity}

ALP activity was assessed via a commercial kit (Nanjing Jiancheng Bioengineering Institute, Jiangsu, China) according to manufacturer's protocols.

\section{Western blotting analysis}

Total proteins were isolated from the whole lysates of cells with cell lysis buffer (Beyotime, Shanghai, China), and from nuclear and cytoplasmic fractions with the Nuclear and Cytoplasmic Protein Extraction Kit (Beyotime, Shanghai, China). The concentrations were analyzed with a BCA Protein Assay Kit (Beyotime). Protein complex was separated by SDS-PAGE, and transferred onto PVDF (Millipore, Bedford, MA, USA) membranes. After blocking with skim milk (5\% M/V), the membranes were incubated with one of the primary antibodies at $4{ }^{\circ} \mathrm{C}$ overnight: anti-CNR2 (1:500; Abclonal, Shanghai, China), anti-Secreted Phosphoprotein 1 (SSP1)/osteopontin (1:500; Proteintech, Wuhan, China), anti-bone gamma-carboxyglutamate (gla) protein (BGLAP)/osteocalcin (1:1000; Abcam, Cambridge, MA, USA), anti-Nrf-2 antibody (1:500; Proteintech), antiLamin B (1:3000; Proteintech), anti-LC3II/I antibody (1: 1000; Cell Signaling Technology, Danvers, MA, USA); anti-Beclin 1 (1:1000; Proteintech), anti-p62 antibody (1: 2000; Proteintech), anti-Keap 1 (1:3000; Proteintech), anti-phosphorylated Mammalian Target Of Rapamycin (p-mTOR; 1:500, CST, Danvers, MA, USA), anti-mTOR (1:1000, CST), anti- phosphorylated Ribosomal Protein S6 Kinase $70 \mathrm{kDa}$ Polypeptide 1 (p-P70S6K; 1:500, CST), anti-p70S6K antibody (1:1000, CST), anti-phosphorylated Eukaryotic Translation Initiation Factor 4E Binding Protein 1 (p-4EBP1; 1:500, Abclonal) and anti-4EBP1 (1: 1000; Affinity, Cincinnati, OH, USA) and GAPDH (1:
10000; Proteintech). Then, the PVDF membranes were incubated with horseradish peroxidase-conjugated goat anti-rabbit secondary antibody (1: 5000; Beyotime) for 45 min at $37^{\circ} \mathrm{C}$. To the end, protein blots were visualized with enhanced chemiluminescence (ECL; Beyotime), and their intensities were analyzed with a gel imaging system. Relative protein expression levels were normalized to GAPDH or Lamin B.

\section{Reverse transcription (RT)-PCR and real-time RT-PCR}

Total RNAs were isolated with TriPure isolation reagent (Bioteke, Beijing, China), and processed into cDNAs via Super M-MLV reverse transcriptase (Bioteke). PCR products were analyzed in agarose gel (1.5\%) electrophoresis. The relative gene expression levels were determined by real-time RT-PCR. In short, cDNA templates were mixed with primers (Genscript, Nanjing, China), Sybr Green (Sigma-Aldrich, St. Louis, MO, USA) and $2 \times$ Power Taq PCR MasterMix (Bioteke), and analyzed on an Exicycler ${ }^{\mathrm{rm}} 96$ PCR instrument. Housekeeping gene GAPDH was used as a reference. Primer information was as following: CNR2-F 5'-agccctcatacctgttcattgg-3'; CNR2-R 5'-gtgaaggtcatagtcacgctg-3'; osteopontin-F, $5^{\prime}$-gaagtttcgcagacctgacat-3', osteopontin-R $5^{\prime}$-gtatgcaccattcaactcctcg-3; osteocalcin-F, 5' - cactcctcgccctattggc $-3^{\prime}$, osteocalcin- $R, 5^{\prime}$-ccctcctgcttggacacaaag- $3^{\prime}$. Data from real-time PCR results were calculated via the $2^{-\Delta \Delta \mathrm{Ct}}$ method.

\section{Immunofluorescent staining}

Cell dishes were washed with $1 \times$ PBS (Sangon, Shanghai, China) for several times, and fixed in $4 \%$ paraformaldehyde (Sinopharm, Shanghai, China) for $15 \mathrm{~min}$ at room temperature. Then, cells were permeabilized with $0.1 \%$ Triton X-100 (Beyotime) at room temperature for 30 $\mathrm{min}$, and blocked with goat serum for $15 \mathrm{~min}$. Thereafter, cell dishes were incubated with anti-Nrf-2 antibody (1:100) or anti-p62 antibody (1:100) overnight at $4{ }^{\circ} \mathrm{C}$. Afterwards, these cells were incubated with Cy3-labled secondary antibody (1:200; Beyotime) for $45 \mathrm{~min}$ at $37^{\circ} \mathrm{C}$. The fluorescent pictures were taken with an Olympus camera (DP73).

\section{Alizarin red staining}

Mineralization of hFOB 1.19 cells were detected with Alizarin red staining. In short, cells seeded in 96-well plates were incubated with $50 \mathrm{nM}$ HU308, $10 \mu \mathrm{M}$ JWH133 or 2 mM 3-MA (MCE, Monmouth Junction, NJ, USA) for $8 \mathrm{~d}$, and fixed in 4\% paraformaldehyde and dyed by Alizarin red S (Solarbio, Beijing, China).

\section{CCK-8 assay}

To determine whether $2 \mathrm{mM} 3-\mathrm{MA}$ treatment affected cell vitality, CCK- 8 assay was performed in hFOB 1.19 
cells cultured at $39^{\circ} \mathrm{C}$ for $96 \mathrm{~h}$ or $192 \mathrm{~h}$. In brief, cells were treated with CCK- 8 reagent (Beyotime) for $1 \mathrm{~h}$, and then the absorption values at $450 \mathrm{~nm}$ were analyzed with a microplate reader.

\section{Statistical analysis}

Values in each histogram were presented as mean \pm standard deviation. Statistical significance was determined with one-way analysis of variance followed by Tukey's analysis (GraphPad Prism 7.0; GraphPad software, La Jolla, CA, USA). Data in Fig. 3 were analyzed with two-way analysis of variance followed by Sidak's analysis (GraphPad Prism 8.0).

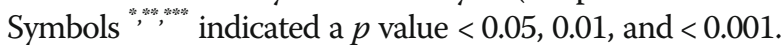

\section{Results}

Expression alterations in CNR2, autophagy molecules, and Nrf2 during osteogenic differentiation in vitro

ALP activities were determined in hFOB 1.19 cells incubated at $39^{\circ} \mathrm{C}$ for $48,96,144$ or 192 h. As seen in Fig. 1a,
ALP activity increased with time. The expression levels of osteopontin and osteocalcin, two osteogenic markers, were analyzed with real-time PCR. Upregulation in these two molecules was observed in differentiated hFOB 1.19 cells (Fig. 1b). Alizarin red staining showed that a 192-h osteoinductive differentiation promoted obvious cell mineralization (Fig. 1c). These data together confirmed that hFOB 1.19 cells had osteogenic differentiation potential at $39{ }^{\circ} \mathrm{C}$.

CNR2 expression was determined with RT-PCR and western blotting. The corresponding data showed that CNR2 mRNA and protein expression levels increased during osteogenic differentiation (Fig. 1d-e). Further, we noted effective autophagy flux in differentiated hFOB 1.19 cells - LC3II/I conversion was enhanced and beclin 1 expression was upregulated, whilst p62 expression was downregulated (Fig. 1f). In addition, less nuclear and more cytoplasmic Nrf2 were detected in differentiated hFOB 1.19 cells (Fig. 1g). These data reveal activation of

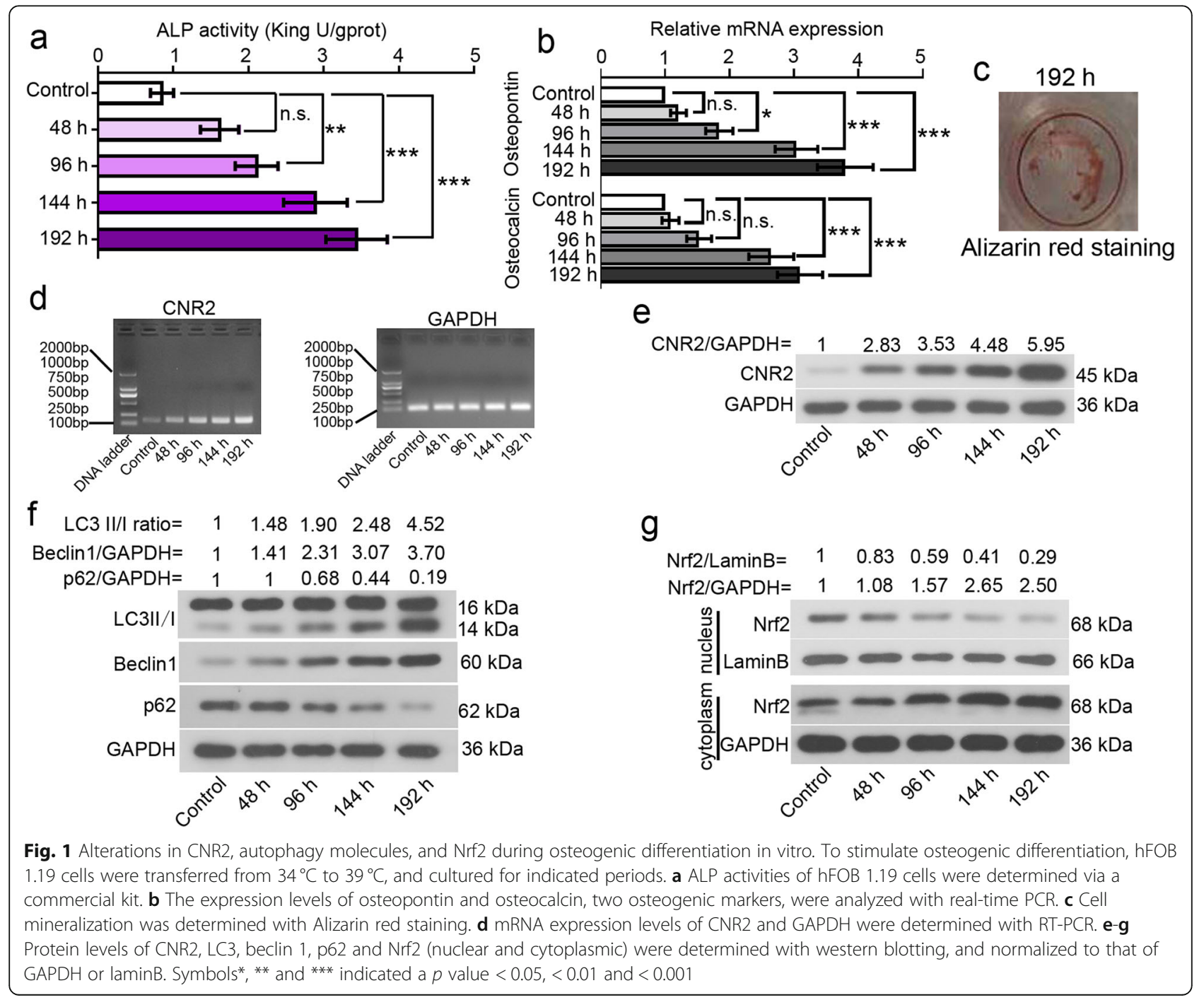


CNR2 and autophagy signaling, and deactivation of Nrf2 signaling during the spontaneous osteogenic differentiation.

\section{CNR2 agonists enhance osteogenic differentiation by activating autophagy}

hFOB 1.19 cells were cultured at $39^{\circ} \mathrm{C}$ for $48 \mathrm{~h}$, and then treated with two CNR2 agonists, HU308 and JWH133, for additional $12 \mathrm{~h}$. Data from western blotting indicated that the CNR2 agonists promoted the conversion of LC3I to LC3II, upregulated beclin 1 expression, and accelerated p62 degradation in hFOB 1.19 cells (Fig. 2a-b). Immunofluorescence images validated the p62 degradation in hFOB 1.19 cells induced by HU308 and JWH133 (Fig. 2c). CNR2 agonists-promoted autophagy was weaken by CNR2 knockdown (Fig. 2d-e). Further, CNR2 agonists suppressed mTOR signaling pathway as evidenced by decreased phosphorylation of mTOR, P70S6K and 4EBP1 in hFOB 1.19 cells (Fig. 2f-g). The signaling transduction of mTOR pathway was partly restored when CNR2 was silenced (Fig. 2f-g). Similar alterations were also observed in human BMMSCs (Additional file 1: Figure S1a-b).

We next blocked cellular autophagy with 3-MA, and evaluated the effects of HU308 and JWH133 on hFOB 1.19 cell osteogenic differentiation. The treatment of 2 mM 3-MA hardly affected cell viability (Additional file 2 : Figure S2). ALP activity significantly increased in cells exposed to HU308 or JWH133, and decreased in response to 3-MA (Fig. 3a). Further, the expression levels of two osteogenic differentiation markers, osteocalcin and osteopontin, were upregulated by CNR2 agonists, and downregulated by 3-MA (Fig. 3b-c). hFOB 1.19 cell mineralization determined with Alizarin red staining was enhanced by CNR2 agonists, and weakened by 3-MA (Fig. 3d). Likewise, 3-MA inhibited CNR2 agonistsinduced osteogenic differentiation of BMMSCs (Additional file 1: Figure S1c-e).

These data together demonstrate that CNR2 agonistsinduced osteogenic differentiation is attenuated when autophagy is hindered.

\section{CNR2 agonists inhibit nuclear Nrf2 expression during osteogenic differentiation}

hFOB 1.19 cells were cultured at $34^{\circ} \mathrm{C}$ until reaching confluence, and then transferred to $39^{\circ} \mathrm{C}$. Forty-eight hours later, cells were exposed to varied concentrations of HU308 and JWH133 for another $12 \mathrm{~h}$. Western blotting results illustrated that CNR2 agonists dosedependently reduced nuclear $\mathrm{Nrf} 2$ accumulation in hFOB 1.19 cells (Fig. 4a-b). Immunofluorescence images validated western blotting results (Fig. 4c).

We determined the expression of CNR2 in hFOB 1.19 cells treated with HU308 or JWH133. Western blotting data indicated that CNR2 agonists induced CNR2 expression in hFOB 1.19 cells (Fig. 4d-e). Further, CNR2 shRNA successfully knocked CNR2 expression down in hFOB 1.19 cells (Fig. 4d-e). CNR2 silencing partly restored Nrf2 signals: nuclear Nrf2 increased and Keap1 expression decreased (Fig. 4d-e). Similar alterations were also observed in human BMMSCs (Additional file 1: Figure S1a-b). The above data suggest that CNR2 agonists trigger the deactivation of Nrf2 signaling pathway in hFOB 1.19 cells.

\section{CNR2 agonists promote p62 degradation, and induce release of Keap1 to retain Nrf2}

P62 is suggested to interact with Keap1-Nrf2 system [17]. In presence of HU308 or JWH133, the protein expression of p62 increased by 5.7- and 4.2-fold in hFOB 1.19 cells post the transfection of SQSTM1 (the gene encoding p62) OE plasmid (Fig. 5a-b). Keap1 expression decreased in response to p62 overexpression (Fig. 5a-b). To determine whether the degradation of Nrf2 was affected by CNR2 agonists and p62, the Nrf2 protein synthesis in hFOB 1.19 cells was first inhibited by $100 \mu \mathrm{g} / \mathrm{ml}$ cycloheximide. As indicated in Fig. 5c, degradation of $\mathrm{Nrf} 2$ protein started at $0.5 \mathrm{~h}(62 \%)$, and reduced to $17 \%$ at $8 \mathrm{~h}$ following cycloheximide treatment. Interestingly, CNR2 agonists significantly augmented Nrf2 degradation in hFOB 1.19 cells. In cells exposed to HU308, Nrf2 degraded to $39 \%$ at $0.5 \mathrm{~h}$, and to $9 \%$ at $8 \mathrm{~h}$ (Fig. 5c). In cells exposed to JWH133, Nrf2 degraded to $51 \%$ at $0.5 \mathrm{~h}$, and to $8 \%$ at $8 \mathrm{~h}$ (Fig. $5 \mathrm{c}$ ). Moreover, CNR2 agonists-induced Nrf2 degradation was markedly attenuated in hFOB 1.19 cells overexpressing p62 (Fig. 5c). For Co-IP assay, beads coated with anti-Keap1 was used to pull down intracellular Keap1, and we found that less Nrf2 bound to Keap1 when p62 was overexpressed (Fig. 5d-e). These data together suggest that CNR2 agonists reduce p62 expression, thereby promoting Nrf2 degradation in hFOB 1.19 cells.

\section{Discussion}

Our prior work has revealed the osteogenesis-promoting effects of CNR2 agonist in BMMSCs [11], and our current work further demonstrates an involvement of autophagy activation in such effects. We find that blockade of autophagy weakens the osteoinductive effects of HU308 and JWH133. CNR2 agonists-induced reduction in p62, an autophagy chaperone, contributes Nrf2 degradation in mature osteoblasts.

Under restrictive temperature $\left(39^{\circ} \mathrm{C}\right)$, hFOB 1.19 cells spontaneously differentiate towards mature osteoblasts [24]. CNR2 expression was previously demonstrated to increase with time in BMMSCs and MC3T3 E1 osteoblastic cells incubated in osteogenic medium [10], and here in differentiated hFOB 1.19 cells. These findings 


\section{a}

LC3 I/II ratio= 11.452 .983 .654 .016 .10

Beclin1/GAPDH= $1 \quad 0.941 .53 \quad 3.05 \quad 4.176 .11$

$\mathrm{p} 62 / \mathrm{GAPDH}=1 \quad \begin{array}{llllll}1 & 0.79 & 0.77 & 0.56 & 0.30 & 0.28\end{array}$

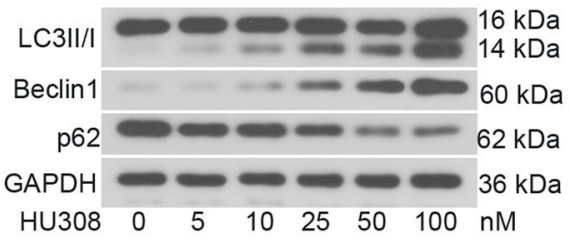

b

LC3 I/II ratio= $1 \quad 1.202 .142 .93 \quad 4.344 .38$

Beclin1/GAPDH= $1 \quad 2.413 .113 .865 .126 .67$

$\mathrm{p} 62 / \mathrm{GAPDH}=1 \quad 0.80 \quad 0.750 .46 \quad 0.25 \quad 0.24$

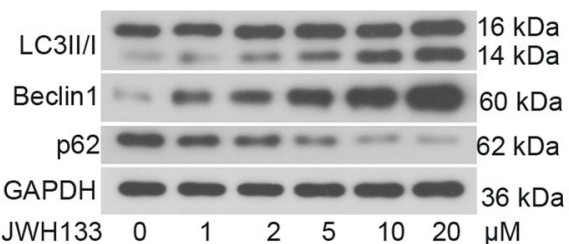

d

C
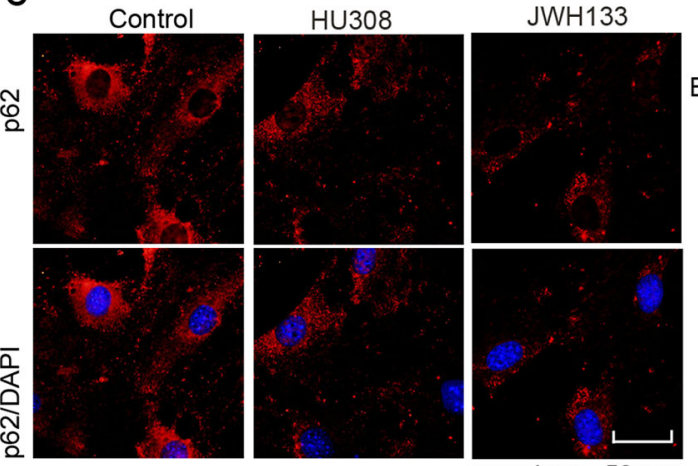

bar $=50 \mu \mathrm{m}$

f

$\mathrm{p}-\mathrm{S} 6 \mathrm{~K} / \mathrm{S} 6 \mathrm{~K}=\begin{array}{llll}1 & 0.09 & 0.08 & 0.46\end{array}$

$\mathrm{p}-\mathrm{mTOR} / \mathrm{mTOR}=\begin{array}{llll}1 & 0.32 & 0.31 & 0.63\end{array}$

p-4E-BP1/4E-BP1 $=\begin{array}{llll}1 & 0.27 & 0.20 & 0.73\end{array}$

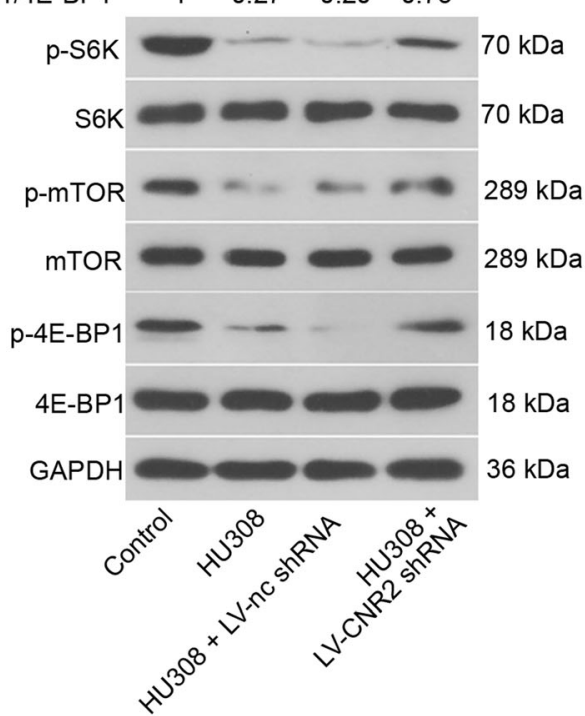

LC3IIIII= 14.512 .14

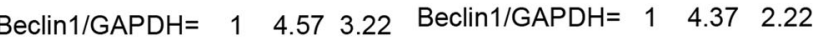

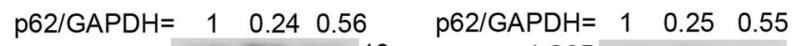

LC3II/I $=16$

Beclin1 -60

p62 -62

GAPDH -36

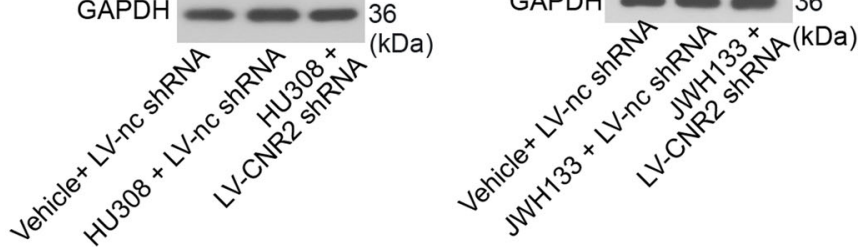

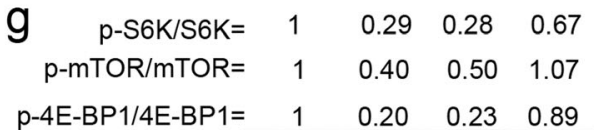

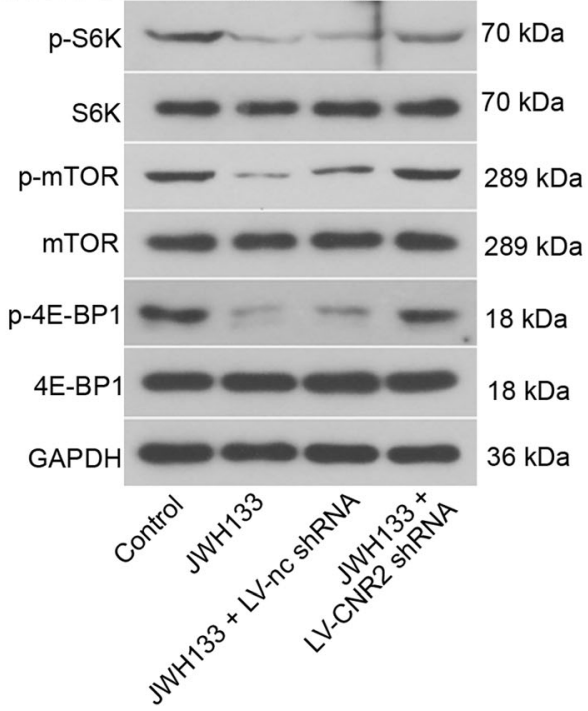

Fig. 2 (See legend on next page.) 
(See figure on previous page.)

Fig. 2 CNR2 agonists activate autophagy and suppress mTOR pathway in hFOB 1.19 cells. hFOB 1.19 cells were cultured at $34^{\circ} \mathrm{C}$ until reaching confluence, and then transferred to $39^{\circ} \mathrm{C}$. Forty-eight hours later, $\operatorname{HU} 308(5,10,25,50$ or $100 \mathrm{nM})$ or JWH133 $(1,2,5,10$ or $20 \mu \mathrm{M})$ was added into the cell medium. Twelve hours later, hFOB 1.19 cells were subjected to $\mathbf{a}-\mathbf{b}$ western blotting analysis. c For immunofluorescence staining of p62, hFOB 1.19 cells were treated with $50 \mathrm{nM} \mathrm{HU308}$ or $10 \mu \mathrm{M}$ JWH133 for $12 \mathrm{~h} . \mathbf{d}-\mathbf{g}$ hFOB 1.19 cells were infected with LV-CNR2 shRNA at $34^{\circ} \mathrm{C}$, and $24 \mathrm{~h}$ later, cells were transferred to $39^{\circ} \mathrm{C}$ for differentiation. Forty-eight hours later, cells were further treated with $50 \mathrm{nM} \mathrm{HU308} \mathrm{or} 10 \mu \mathrm{MM}$ JWH133 for $12 \mathrm{~h}$, and then harvested for western blotting analysis

suggest that CNR2 elevation is associated with enhanced osteogenic differentiation. Interestingly, we noted that the basal autophagy of hFOB 1.19 cells was enhanced during the spontaneous differentiation period. Conversion of LC3I to LC3II was augmented, beclin1 expression was upregulated, and most importantly, p62 degraded significantly.

As an autophagy adaptor, p62 mediates the ubiquitin degradation of LC3 protein aggregates, and p62 itself is degraded by autophagy [25]. Thus, the successful

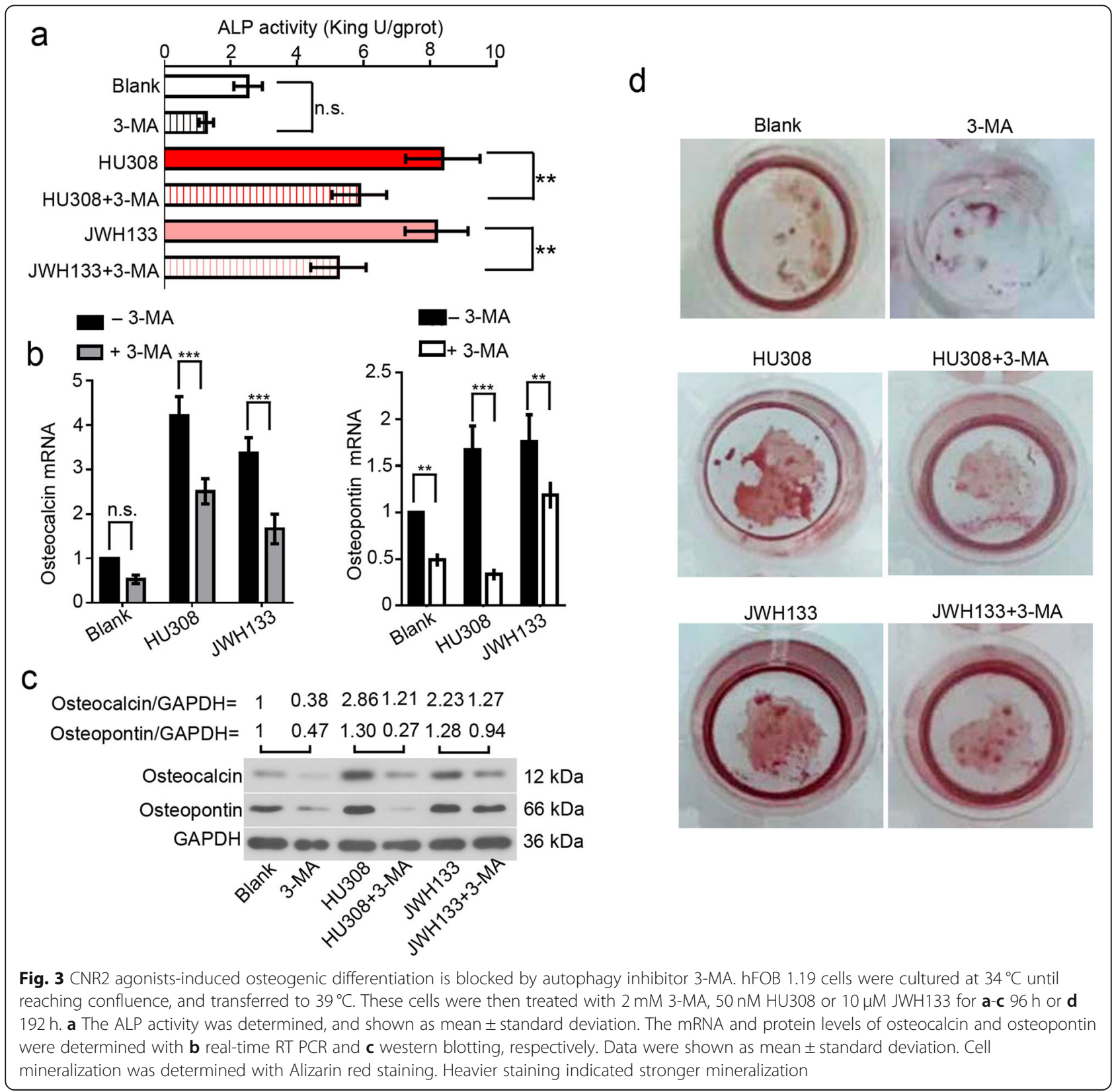




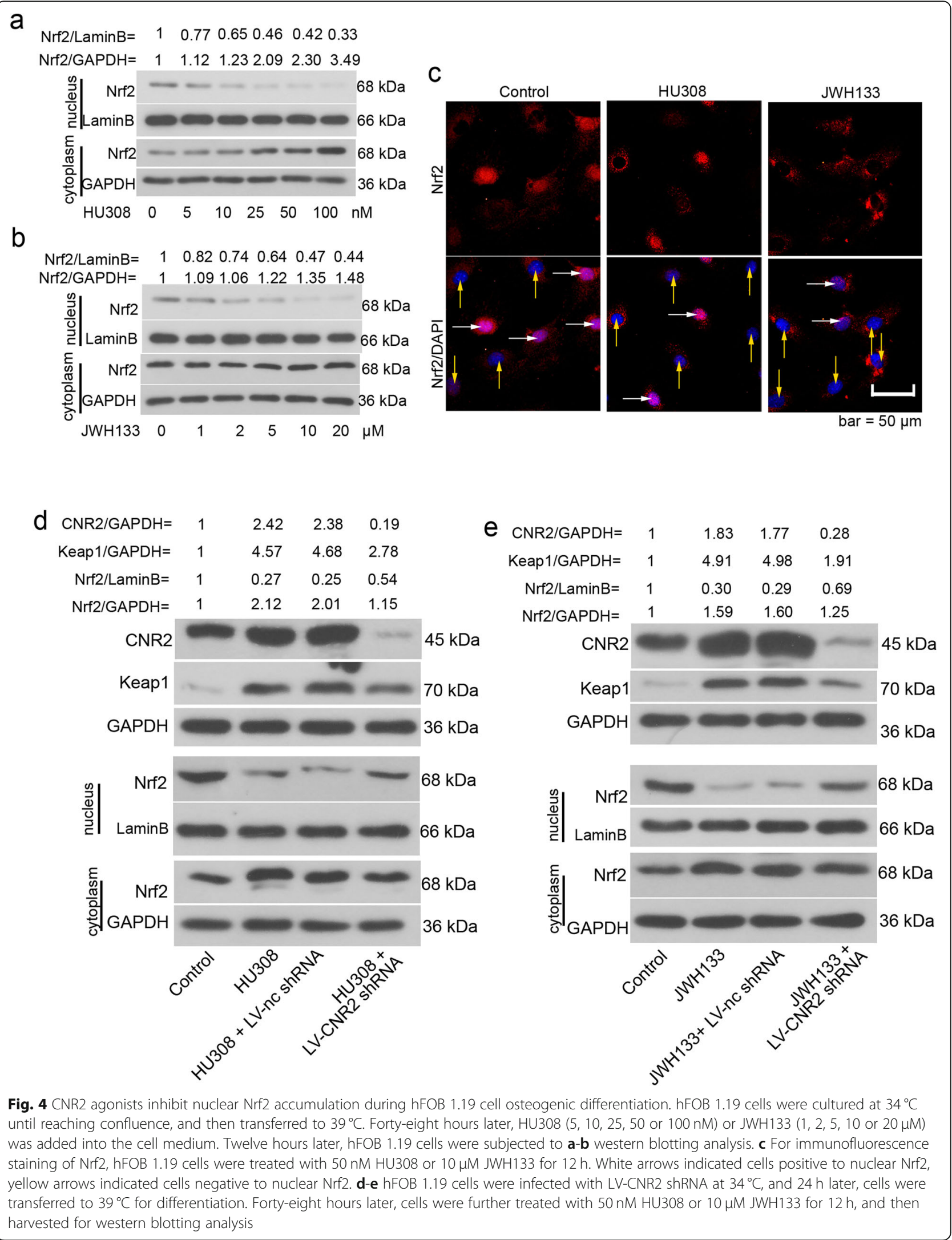




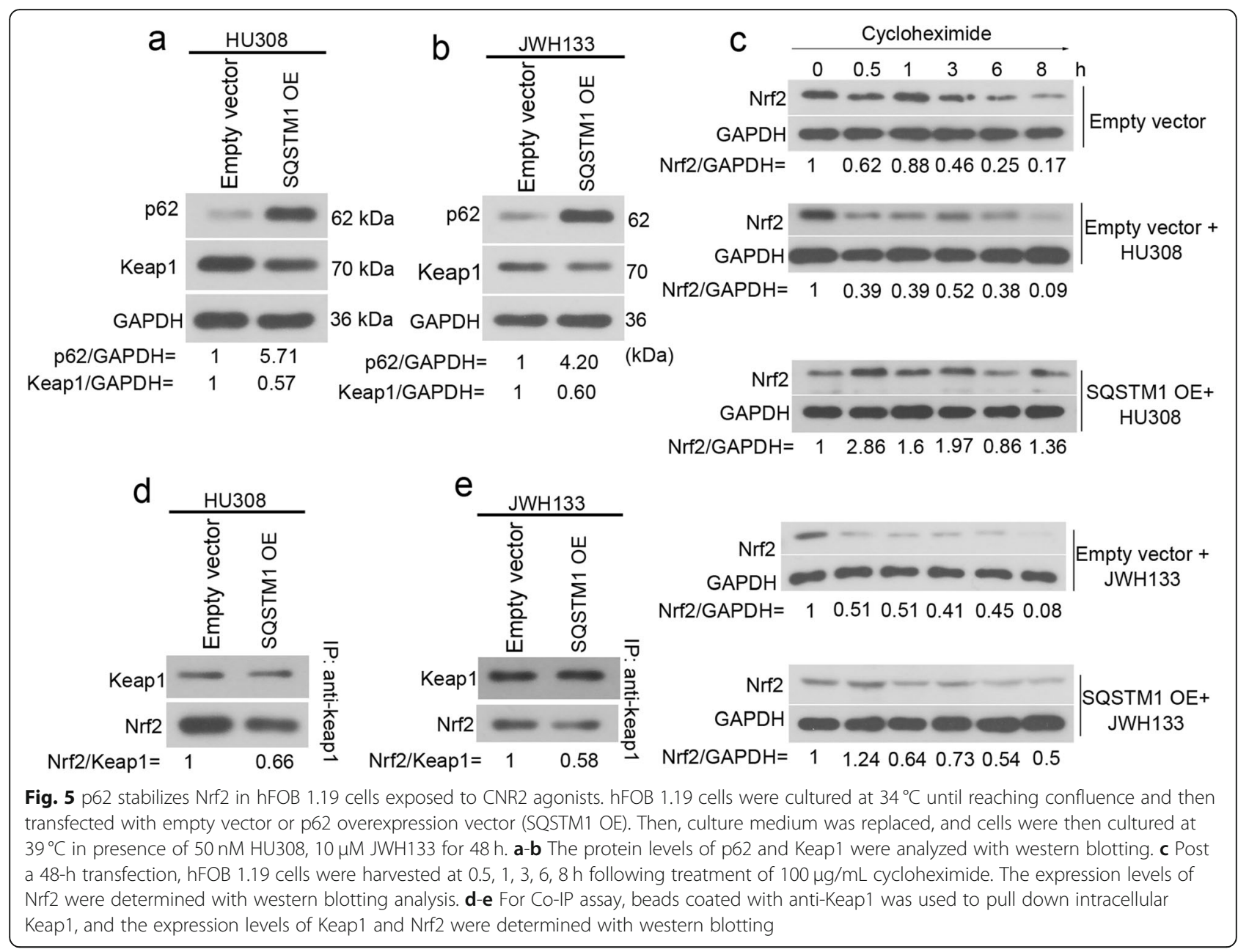

degradation of p62 indicates an effective autophagic flux in differentiated hFOB 1.19 cells. On the basis of previous studies showing that CNR2 agonist HU308 augmented cellular autophagy $[14,15]$, we propose that CNR2 activation contributes to autophagy initiation in the differentiated osteoblasts. To valid this hypothesis, we treated hFOB 1.19 cells with increasing doses of CNR2 ligands HU308 and JWH133, and found that the autophagic flux was gradually enhanced. mTOR, a serine/threonine protein kinase, is a core component of mTOR complex 1 (mTORC1), the activation of which potently inhibits autophagy [26, 27]. Administration of mTOR inhibitor (rapamycin) or genetic ablation of mTOR has been reported to preserve the function of osteoblasts and osteocytes, and prevent bone loss [28, 29]. In addition to initiating autophagy, we also found that both CNR2 agonists suppressed mTOR signaling transduction. The inhibitory effects of HU308 on mTOR pathway in cardiomyocytes observed by Wu et al. [15] indirectly supported our current findings. Furthermore, CNR2 ligands' effects on mTOR associated-autophagy were attenuated in CNR2-silenced cells. These data confirm that the activation of CNR2 signaling triggers autophagy in osteoblastic cells.

To explore how the autophagy activated by CNR2 agonists affects osteoblast differentiation, 3-MA was used to block autophagy in hFOB 1.19 cells. Interestingly, we found that CNR2 agonists-induced bone formation in vitro was attenuated when autophagy was blocked with 3-MA. These data suggest that autophagy induction is required for CNR2-medidated osteoblastic differentiation.

Bone formation is a dynamic process that is orchestrated by the balance between osteoblasts and osteoclasts [30]. Besides osteoblasts, the bone resorbing osteoclasts also generate endocannabinoids and express CNR2 [30]. While we demonstrate the osteogenesispromoting effects of CNR2 agonists, others prove their osteoclastogenesis-promoting effects [8]. The opposite results revealing CNR2's role in bone formation in osteoporotic animals $[8,10]$ may attribute to the enhanced differentiation of both bone formatting (osteoblasts) and absorbing (osteoclasts) cell lineages upon CNR2 activation. Furthermore, inhibition of autophagy 
with chloroquine suppressed osteoclast formation induced by receptor activator of nuclear factor kappa-B ligand (RANKL) [31]. This finding along with ours suggest that autophagy is involved not only in CNR2regulated osteoblastic differentiation, but also in osteoclastic differentiation. Designing a cannabinoid-based anti-osteoporotic therapy should consider the dual role of CNR2 signaling in promoting osteoblast and osteoclast differentiation.

Oxidative stress acts a negative contributor to bone formation by inhibiting osteoblast differentiation, in which the Keap1-Nrf2 signaling plays an important role [32]. During the spontaneous osteogenic differentiation, we found that the basal expression of Nrf2 decreased in hFOB 1.19 cells. Adipose derived stem cells incubated in osteogenic medium also had reduced expression of Nrf2 [33]. This earlier study along with our present findings suggests that osteoblastic differentiation requires Nrf2 deactivation. Since CNR2 showed an opposite expression pattern to Nrf2 in hFOB 1.19 cells during differentiation, we assumed that CNR2 negatively regulated Nrf2 signaling transduction in osteoblasts. By further treating hFOB 1.19 cells with HU308 and JWH133, we noted that Nrf2 expression was downregulated, and that Keap1, a molecule known to mediate Nrf2 degradation [16, 17], was upregulated. Given to the negative role of over-activated Nrf2 signaling in regulating osteoblast differentiation [32], our results suggest that CNR2 activation-induced bone formation in vitro is associated with Nrf2 deactivation. $\mathrm{Li}$ and co-workers treated primarily cultured cardiac fibroblasts with another CNR2 agonist, AM1241, and found that, under oxygen and glucose deprivation condition, CNR2 activation further activated Nrf2 signaling transduction [34]. Such inconsistent result is from a total different cell lineage, which hardly disproves our current findings.

P62 stays in the center of autophagy and Keap1-Nrf2 system interaction [35]. Containing a KIR enables p62 to inactivate Keap1, thereby promoting Nrf2 activation [23]. As p62 degradation was induced by CNR2 ligands in hFOB 1.19 cells, additional experiments were performed to determine p62's effects on Nrf2 expression in osteoblasts. Protein synthesis of hFOB 1.19 cells was blocked with cycloheximide, and the Nrf2 protein stabilization was determined. Interestingly, both HU308 and JWH133 accelerated Nrf2 degradation in hFOB 1.19 cells, which was prevented by forced overexpression of p62. These data reveal that CNR2 destabilizes Nrf2 by inducing p62 degradation in differentiated osteoblasts. As Nrf2 degradation is enhanced, it is predictable that more Keap1 binds to Nrf2 and leads to its ubiquitination mediated by ubiquitin E3 ligase complex. Nonetheless, to confirm this assumption, additional experiments detecting the ubiquitination of Nrf2 is needed.
hFOB 1.19 is an osteoblastic cell line established by transfection of temperature sensitive SV40 T-antigen. It has minimal chromosome abnormalities [24, 36], and thus is used as the in vitro model for studying the osteogenic differentiation. However, osteoblasts are actually derived from BMMSCs [37]. Hence, we further validated our major findings in human BMMSCs. Our data demonstrate an involvement of autophagy and Keap1Nrf2 axis in CNR2-mediated osteoblastic differentiation only on the basis of data from the in vitro experiments. Using glucocorticoids- or ovariectomy-induced osteoporosic animal models will help to draw a more valid conclusion.

\section{Conclusion}

Our work shows that CNR2 activation-induced osteoblastic differentiation in vitro is associated with autophagy induction and p62-mediated Nrf2 deactivation. These findings add novel insights into how CNR2mediated cannabinoid signaling contributes to bone homeostasis.

\section{Supplementary information}

Supplementary information accompanies this paper at https://doi.org/10. 1186/s12964-020-0512-6.

Additional file 1: Figure S1. CNR2 agonists induce osteogenic differentiation and promote autophagy of human BMMSCs. To determine alterations in autophagy-associated molecules and Nrf2 signals, BMMSCs were infected with LV-CNR2 shRNA or control lentiviruses, and $24 \mathrm{~h}$ later, they were cultured in osteoinductive media for $48 \mathrm{~h}$, and then treated with $50 \mathrm{nM} \mathrm{HU308}$ or $10 \mu \mathrm{M}$ JWH133 for $12 \mathrm{~h}$. (a-b) The protein levels of indicated molecules were determined with western blotting analysis. BMMSCs were incubated in osteoinductive media in presence of $50 \mathrm{nM}$ HU308, $10 \mu \mathrm{M}$ JWH133 or 2 mM 3-MA. (c) The ALP activities and (d) the mRNA expression of osteopontin and osteocalcin of BMMSCs were determined after a 2-wk culture. (e) Cell mineralization was determined with Alizarin red staining after a 3 -wk culture. Symbols ** and ${ }^{* *}$ indicated a $p$ value $<0.01$ and $<0.001$

Additional file 2: Figure S2. 3-MA treatment hardly affects the vitality of hFOB 1.19 cells. hFOB 1.19 cells were cultured at $34^{\circ} \mathrm{C}$ until reaching confluence, and then transferred to $39^{\circ} \mathrm{C}$. hFOB 1.19 cells were cultured at $34^{\circ} \mathrm{C}$ until reaching confluence, and transferred to $39^{\circ} \mathrm{C}$. These cells were then treated with 2 mM 3-MA, 50 nM HU308 or 10 MM JWH133 for (a) $96 \mathrm{~h}$ or (b) $192 \mathrm{~h}$, and their vitalities were determined with CCK8 assay.

\section{Abbreviations}

4EBP1: Eukaryotic Translation Initiation Factor 4E Binding Protein 1; ALP: Alkaline phosphatase; BGLAP/osteocalcin: Bone gammacarboxyglutamate (gla) protein; CNR2: Cannabinoid receptor type 2; KEAP1: Kelch Like ECH Associated Protein 1; mTOR: Mammalian Target Of Rapamycin; Nrf2: Nuclear Factor, Erythroid 2 Like 2; P70S6K: Ribosomal Protein S6 Kinase 70 kDa Polypeptide 1; RNAi: RNA interference; SQSTM1/ p62: Sequestosome 1; SSP1/osteopontin: Secreted Phosphoprotein 1

\section{Acknowledgements}

None.

Authors' contributions

$A X$ and $Y S$ conceived and designed the study. AX, YY, YS, and MW conducted experiments. $A X$ and $Y Y$ analyzed data. AX wrote the manuscript. All authors read and approved the final manuscript. 


\section{Funding}

This study was supported by a grant from the National Natural Science Foundation of China (No. 81572221).

\section{Availability of data and materials}

All data generated or analyzed during this study are included in this published article.

\section{Ethics approval and consent to participate}

Not applicable.

\section{Consent for publication}

Not applicable.

\section{Competing interests}

The authors declare that they have no competing interests.

Received: 10 June 2019 Accepted: 4 January 2020

Published online: 15 January 2020

\section{References}

1. Pisani P, Renna MD, Conversano F, Casciaro E, Di Paola M, Quarta E, Muratore M, Casciaro S. Major osteoporotic fragility fractures: risk factor updates and societal impact. World J Orthop. 2016;7:171-81.

2. Rahman P, Gladman DD, Urowitz MB. Smoking interferes with efficacy of antimalarial therapy in cutaneous lupus. J Rheumatol. 1998;25:1716-9.

3. Rizzoli R, Biver E. Glucocorticoid-induced osteoporosis: who to treat with what agent? Nat Rev Rheumatol. 2015;11:98-109.

4. Lewiecki EM. New targets for intervention in the treatment of postmenopausal osteoporosis. Nat Rev Rheumatol. 2011;7:631-8.

5. Marie PJ. Osteoblast dysfunctions in bone diseases: from cellular and molecular mechanisms to therapeutic strategies. Cell Mol Life Sci. 2015;72: 1347-61.

6. Corrado A, Sanpaolo ER, Di Bello S, Cantatore FP. Osteoblast as a target of anti-osteoporotic treatment. Postgrad Med. 2017;129:858-65.

7. Rhee MH, Vogel Z, Barg J, Bayewitch M, Levy R, Hanus L, Breuer A, Mechoulam R. Cannabinol derivatives: binding to cannabinoid receptors and inhibition of adenylylcyclase. J Med Chem. 1997;40:3228-33.

8. Idris Al, Sophocleous A, Landao-Bassonga E, van't Hof RJ, Ralston SH. Regulation of bone mass, osteoclast function, and ovariectomy-induced bone loss by the type 2 cannabinoid receptor. Endocrinology. 2008;149: 5619-26.

9. Sophocleous A, Marino S, Kabir D, Ralston SH, Idris Al. Combined deficiency of the $\mathrm{Cnr} 1$ and $\mathrm{Cnr} 2$ receptors protects against age-related bone loss by osteoclast inhibition. Aging Cell. 2017;16:1051-61.

10. Ofek O, Karsak M, Leclerc N, Fogel M, Frenkel B, Wright K, Tam J, AttarNamdar M, Kram V, Shohami E, et al. Peripheral cannabinoid receptor, CB2, regulates bone mass. Proc Natl Acad Sci U S A. 2006;103:696-701.

11. Sun $Y X, X u A H$, Yang $Y$, Zhang JX, Yu AW. Activation of cannabinoid receptor 2 enhances osteogenic differentiation of bone marrow derived mesenchymal stem cells. Biomed Res Int. 2015;2015:874982.

12. Klionsky DJ, Emr SD. Autophagy as a regulated pathway of cellular degradation. Science. 2000;290:1717-21

13. Qi M, Zhang L, Ma Y, Shuai Y, Li L, Luo K, Liu W, Jin Y. Autophagy maintains the function of bone marrow Mesenchymal stem cells to prevent estrogen deficiency-induced osteoporosis. Theranostics. 2017;7:4498-516.

14. Ke P, Shao BZ, Xu ZQ, Wei W, Han BZ, Chen XW, Su DF, Liu C. Activation of cannabinoid receptor 2 ameliorates DSS-induced colitis through inhibiting NLRP3 Inflammasome in macrophages. PLoS One. 2016:11:e0155076.

15. Wu A, Hu P, Lin J, Xia W, Zhang R. Activating cannabinoid receptor 2 protects against diabetic cardiomyopathy through autophagy induction. Front Pharmacol. 2018;9:1292.

16. Villeneuve NF, Lau A, Zhang DD. Regulation of the Nrf2-Keap1 antioxidant response by the ubiquitin proteasome system: an insight into cullin-ring ubiquitin ligases. Antioxid Redox Signal. 2010;13:1699-712.

17. Taguchi K, Motohashi H, Yamamoto M. Molecular mechanisms of the Keap1-Nrf2 pathway in stress response and cancer evolution. Genes Cells. 2011;16:123-40.

18. Yoshida E, Suzuki T, Morita M, Taguchi K, Tsuchida K, Motohashi H, Doita M, Yamamoto M. Hyperactivation of Nrf2 leads to hypoplasia of bone in vivo. Genes Cells. 2018;23:386-92.
19. Hinoi E, Fujimori S, Wang L, Hojo H, Uno K, Yoneda Y. Nrf2 negatively regulates osteoblast differentiation via interfering with Runx2-dependent transcriptional activation. J Biol Chem. 2006;281:18015-24.

20. Bjorkoy G, Lamark T, Brech A, Outzen H, Perander M, Overvatn A, Stenmark H, Johansen T. p62/SQSTM1 forms protein aggregates degraded by autophagy and has a protective effect on huntingtin-induced cell death. J Cell Biol. 2005;171:603-14.

21. Donaldson KM, Li W, Ching KA, Batalov S, Tsai CC, Joazeiro CA. Ubiquitinmediated sequestration of normal cellular proteins into polyglutamine aggregates. Proc Natl Acad Sci U S A. 2003;100:8892-7.

22. Taillebourg E, Gregoire I, Viargues $\mathrm{P}$, Jacomin AC, Thevenon D, Faure M, Fauvarque MO. The deubiquitinating enzyme USP36 controls selective autophagy activation by ubiquitinated proteins. Autophagy. 2012:8:767-79.

23. Komatsu M, Kurokawa H, Waguri S, Taguchi K, Kobayashi A, Ichimura Y, Sou YS, Ueno I, Sakamoto A, Tong Kl, et al. The selective autophagy substrate p62 activates the stress responsive transcription factor Nrf2 through inactivation of Keap1. Nat Cell Biol. 2010;12:213-23.

24. Harris SA, Enger RJ, Riggs BL, Spelsberg TC. Development and characterization of a conditionally immortalized human fetal osteoblastic cell line. J Bone Miner Res. 1995:10:178-86.

25. Pankiv S, Clausen TH, Lamark T, Brech A, Bruun JA, Outzen H, Overvatn A, Bjorkoy G, Johansen T. p62/SQSTM1 binds directly to Atg8/LC3 to facilitate degradation of ubiquitinated protein aggregates by autophagy. J Biol Chem. 2007:282:24131-45

26. Kim YC, Guan KL. mTOR: a pharmacologic target for autophagy regulation. J Clin Invest. 2015:125:25-32

27. Johnson CE, Tee AR. Exploiting cancer vulnerabilities: mTOR, autophagy, and homeostatic imbalance. Essays Biochem. 2017:61:699-710.

28. Chagin AS. Effectors of mTOR-autophagy pathway: targeting cancer, affecting the skeleton. Curr Opin Pharmacol. 2016;28:1-7.

29. Tanaka Y, Sonoda S, Yamaza H, Murata S, Nishida K, Hama S, KyumotoNakamura Y, Uehara N, Nonaka K, Kukita T, Yamaza T. Suppression of AKTmTOR signal pathway enhances osteogenic/dentinogenic capacity of stem cells from apical papilla. Stem Cell Res Ther. 2018;9:334

30. Bab I, Zimmer A. Cannabinoid receptors and the regulation of bone mass. Br J Pharmacol. 2008;153:182-8

31. Xiu Y, Xu H, Zhao C, Li J, Morita Y, Yao Z, Xing L, Boyce BF. Chloroquine reduces osteoclastogenesis in murine osteoporosis by preventing TRAF3 degradation. J Clin Invest. 2014;124:297-310.

32. Wauquier $F$, Leotoing $L$, Coxam V, Guicheux J, Wittrant $Y$. Oxidative stress in bone remodelling and disease. Trends Mol Med. 2009;15:468-77.

33. Tao J, Wang H, Zhai Y, Park H, Wang J, Ji F, Zhang Z. Downregulation of Nrf2 promotes autophagy-dependent osteoblastic differentiation of adipose-derived mesenchymal stem cells. Exp Cell Res. 2016:349:221-9.

34. Li X, Han D, Tian Z, Gao B, Fan M, Li C, Li X, Wang Y, Ma S, Cao F. Activation of cannabinoid receptor type II by AM1241 ameliorates myocardial fibrosis via Nrf2-mediated inhibition of TGF-beta1/Smad3 pathway in myocardial infarction mice. Cell Physiol Biochem. 2016:39:1521-36.

35. Ichimura Y, Waguri S, Sou YS, Kageyama S, Hasegawa J, Ishimura R, Saito T, Yang Y, Kouno T, Fukutomi T, et al. Phosphorylation of p62 activates the Keap1-Nrf2 pathway during selective autophagy. Mol Cell. 2013;51:618-31.

36. Subramaniam M, Jalal SM, Rickard DJ, Harris SA, Bolander ME, Spelsberg TC. Further characterization of human fetal osteoblastic hFOB 1.19 and hFOB/ER alpha cells: bone formation in vivo and karyotype analysis using multicolor fluorescent in situ hybridization. J Cell Biochem. 2002:87:9-15.

37. Aubin JE. Regulation of osteoblast formation and function. Rev Endocr Metab Disord. 2001:2:81-94.

\section{Publisher's Note}

Springer Nature remains neutral with regard to jurisdictional claims in published maps and institutional affiliations. 